Article

\title{
Evaluation of a Spatial Heterodyne Spectrometer for Raman Spectroscopy of Minerals
}

\author{
Alina Zettner ${ }^{1}$, Ardian B. Gojani ${ }^{1, *} \mathbb{C}$, Thomas Schmid ${ }^{1,2}$ and Igor B. Gornushkin $^{1}$ \\ 1 BAM Federal Institute of Materials Research and Testing, Richard-Willstäter-Strasse 11, 12489 Berlin, \\ Germany; alinazettner@aol.com (A.Z.); thomas.schmid@bam.de (T.S.); igor.gornushkin@bam.de (I.B.G.) \\ 2 School of Analytical Sciences Adlershof (SALSA), Humboldt-Universität zu Berlin, Unter den Linden 6 , \\ 10099 Berlin, Germany \\ * Correspondence: ardian.gojani@bam.de
}

Received: 30 January 2020; Accepted: 20 February 2020; Published: 24 February 2020

\begin{abstract}
Spatial heterodyne spectroscopy (SHS) is a novel spectral analysis technique that is being applied for Raman spectroscopy of minerals. This paper presents the theoretical basis of SHS and its application for Raman measurements of calcite, quartz and forsterite in marble, copper ore and nickel ore, respectively. The SHS measurements are done using a broadband $(518-686 \mathrm{~nm})$ and resolving power $R \approx 3000$ instrument. The spectra obtained using SHS are compared to those obtained by benchtop and modular dispersive spectrometers. It is found that SHRS performance in terms of resolution is comparable to that of the benchtop spectrometer and better than the modular dispersive spectrometer, while the sensitivity of SHRS is worse than that of a benchtop spectrometer, but better than that of a modular dispersive spectrometer. When considered that SHS components are small and can be packaged into a handheld device, there is interest in developing an SHS-based instrument for mobile Raman spectroscopy. This paper evaluates the possibility of such an application.
\end{abstract}

Keywords: spatial heterodyne spectrometer; interferometric spectroscopy; fourier transform spectroscopy; Raman spectroscopy; calcite; quartz; forsterite

\section{Introduction}

Raman spectroscopy is recognized as a powerful method for material characterization, therefore, the development of novel instrumentation for Raman spectroscopy is an active area of research. Among that, spatial heterodyne (Raman) spectroscopy (SHS or SHRS) has attracted attention thanks to its promise of increased sensitivity, robustness, small size of the instrument and low cost. The modern development of SHS was motivated by astronomical research [1], but, since the first application of SHS to Raman spectroscopy [2,3], a number of laboratories have applied the technique for chemical analysis of various materials, including minerals: rose quartz and celestine [2]; quartz [3]; forsterite, plagioclase, microcline, dolomite, calcite, gypsum, and anhydrite [4]; calcite [5]; gypsum, quartz, calcite in rocks, the latter also in a, snail shell [6], to name a few.

SHS is an interferometry-based spectrometer and it is a modification of the Michelson interferometer, where the mirrors (scanning and fixed) are substituted by stationary diffraction gratings. The main attractiveness of SHS lies in its simultaneous high resolution, broad spectral range, and the high throughput of the instrument. The latter is a feature of all interferometers, but SHS does not require scanning, as Michelson interferometers, nor is it limited to the IR region, as FTIR instruments are. On the other hand, SHS is a demanding instrument with regards to data collection and treatment, because the result of its measurement is the interferogram, which must be converted to a conventional spectrum with the intensity vs. the wavelength. 
Because they are able to deliver information on both, inorganic and organic constituents of samples, portable Raman devices have great potential for applications in gemmology, geological field studies, drill core logging, and hydrocarbon prospection. Due to their small size and robustness, spatial heterodyne Raman spectrometers are good candidates for such applications. Indeed, spatial heterogeneity and complex compositions of according samples are demanding, and fluorescence emission by either organic sample constituents or luminescent minerals are typical interferences in Raman spectra in general, which hamper some applications. Nevertheless, if a Raman device can be technically adapted to a certain application, e.g., by using an optimized excitation wavelength, such issues might be reduced, and a spatial heterodyne Raman spectrometer employing an ultraviolet laser source has already been demonstrated to enable blocking of the major part of long-wavelength fluorescence emission by optical filters [6].

Spatial heterodyne Raman spectrometers are characterized by high level of customizability and the user can choose parameters that suit the application the best. For example, SHRS was applied for investigation of celestine, calcite, and rose quartz with two different modifications: in one case, an echelle grating was used [7], and in the other, the gratings were complemented by field-widening wedges in order to increase the sensitivity of SHRS [8]. The current paper gives details of the working principle of SHRS and describes its application for mineralogical characterization of rocks as a proof of principle for future use in compact and mobile devices. The performance of the SHRS instrument in our laboratory is compared to that of a miniature fiber optic dispersive spectrometer, because both these instruments can be used for field measurements. Recently, SHRS has been constructed into a monolithic device with linear dimension about $35 \mathrm{~mm}$ and weight less than $100 \mathrm{~g}$, which can readily be used in hand-held instruments or on-board of weight or size demanding spacecrafts [9].

\section{Spatial Heterodyne Spectroscopy}

The basic principle of operation of SHS is illustrated in Figure 1. An incident plane wave is split by the beamsplitter $B S$ and illuminates each of the diffraction gratings $G_{1}$ and $G_{2}$. Initially, the gratings are placed perpendicular to the optical axis, with the grooves parallel to the $y$ axis (normally to the plane of the page), and then one of the gratings is slightly tilted (by pitch rotation), to make an angle $\eta$ between the plane of the grating and the $y$ axis. This is the configuration for the conical diffraction, instead of the common in-plane diffraction. The incident plane wave is characterized by either wavelength $\lambda$ or wave number $\sigma$, which are related by $\sigma=1 / \lambda$. Different polychromatic wavefronts will be diffracted at angles $\beta(\sigma)$, according to the diffraction grating equation [10]

$$
\cos \eta(\sin \beta+\sin \theta)=\frac{m}{d} \frac{1}{\sigma}
$$

where $m$ is the diffraction order, $d$ is the groove spacing, and $\theta$ is the angle between the grating normal and the optical axis.

For a specific setup, $m$ and $d$ are constants and in practice the beamsplitter produces two waves equal in every respect, except for the direction of propagation. The gratings are rotated for the angle $\theta$, such that the dispersed light reaches the detector. The detector then records the interference pattern of two plane waves propagating in different directions. For a monochromatic wavefont, these directions are noted by wave vectors $\vec{k}_{1}$ and $\vec{k}_{2}$, as shown in Figure 2 . The apparent origin of these vectors is on the plane of the gratings-that is the plane of localization for the interference fringes. This means that the imaging lenses should focus on the gratings and image them onto the detector. 


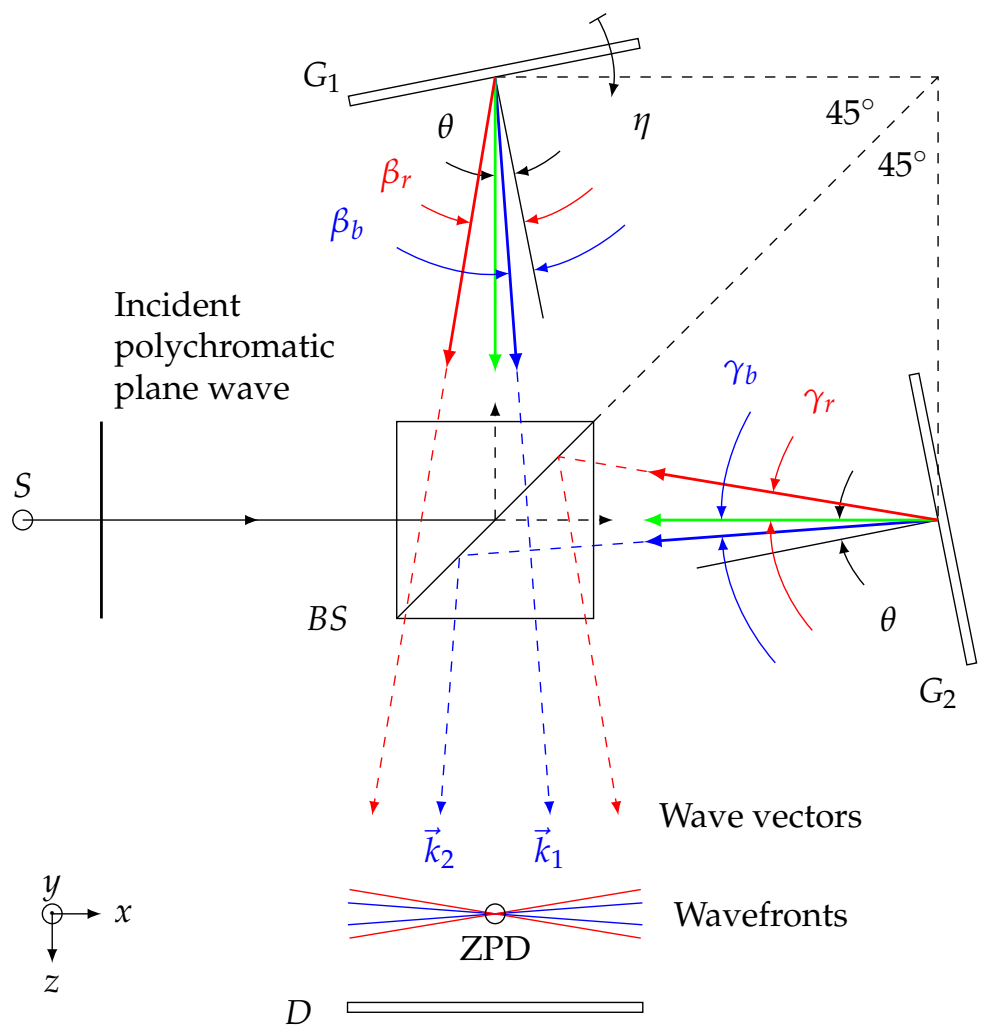

Figure 1. Illustration of the basic principle of spatial heterodyne spectroscopy (SHS): $S$ is the light source, $B S$ is the cube beam splitter, $G_{1}$ and $G_{2}$ are the gratings, rotated by the angle $\theta$ with regards to the optical axes, $D$ is the detector plane, and ZPD is the zero-path-difference line (this line is parallel to the grooves in the grating, i.e., axis $y$ ), and wave vectors $\vec{k}_{1}$ and $\vec{k}_{2}$ for an incident monochromatic wavefront, as seen from the detector. Grating $G_{1}$ is tilted for a very small angle $\eta$ with respect to the $y$ axis. Note that the directions of $\gamma_{r}=\beta_{r}-\theta$ and $\gamma_{b}=\beta_{b}-\theta$ are opposite, designating that these angles-which correspond to wavefronts on the opposite side of the spectrum with regards to the wavefront at Littrow configuration-have different signs. Lenses that collimate the light from the source and those that image the interference fringes on the detector are not shown.

Let us assume that the diffracted wavefronts have coherence lengths larger than the detector size. The interference of these waves on the detector plane will yield the irradiance modulation described by [11]

$$
I \propto 1+\cos \left[\vec{r} \cdot\left(\vec{k}_{1}-\vec{k}_{2}\right)\right] .
$$

Since $k=\left|\vec{k}_{1}\right|=\left|\vec{k}_{2}\right|=2 \pi \sigma$ holds, we obtain

$$
\vec{r} \cdot\left(\vec{k}_{1}-\vec{k}_{2}\right)=4 \pi \sigma x \sin \gamma+2 \pi \sigma y \sin \eta,
$$

where $\gamma=\beta-\theta$ is the angle that the diffracted wavefront with wave number $\sigma$ makes with the optical axis. The consideration of very long coherence length means that $z \cdot\left(k_{1 z}-k_{2 z}\right)=0$ for any $z$.

The special case $\gamma=0$ and $\eta=0$ defines the Littrow configuration of the interferometer and determines the wave number $\sigma_{0}$ of the wavefront that is reflected back in the same direction as the incident wavefront and produces no interference fringes. For this wavefront, based on Equation (1),

$$
\sigma_{0}=\frac{m}{2 d} \frac{1}{\sin \theta} .
$$

All other wavefronts will be exiting the interferometer and reach the detector at exit angles $\pm \gamma$, where plus and minus signs correspond to the first and second grating, correspondingly. (Note: there is 
a change of sign of the angle $\gamma$ due to the reflection of only one wavefront in the direction of the detector from the beamsplitter.) Using the small angle approximation, angle $\gamma$ can be expressed as a function of any wave number $\sigma$ by substituting Equation (4) into Equation (1), from where

$$
\gamma= \pm 2 \tan \theta\left(1-\frac{\sigma_{0}}{\sigma}\right) .
$$

Hence, Equation (3) is expressed as

$$
\vec{r} \cdot\left(\vec{k}_{1}-\vec{k}_{2}\right)=2 \pi\left[4 \tan \theta \cdot\left(\sigma-\sigma_{0}\right) \cdot x+\eta \cdot \sigma \cdot y\right] .
$$

Substitution of Equation (6) into Equation (2), and considering the overall irradiance on the detector caused by the polychromatic light with spectral density $B(\sigma)$, we obtain the interferogram

$$
I(\xi, \zeta)=\int_{-\infty}^{\infty} B(\sigma)\left(1+\cos 2 \pi\left[\xi\left(\sigma-\sigma_{0}\right)+\zeta \sigma\right]\right) d \sigma
$$

where $\xi=4 \tan \theta \cdot x, \zeta=\eta \cdot y$, and the lower limit of the integral can be extended to $-\infty$ because $B(\sigma)=0$ for $\sigma<0$. This interferogram presents the heterodyned about Littrow wave number Fourier transform of the input spectrum $B(\sigma)$ along the coordinate $\xi=\xi(x)$ and nonheterodyned transform along the coordinate $\zeta=\zeta(y)$.

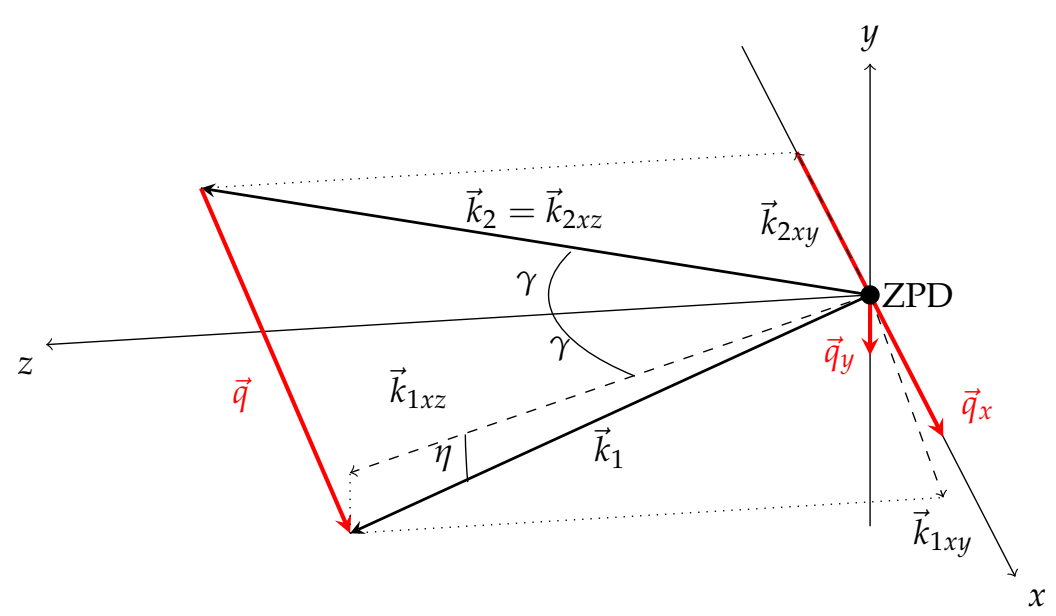

Figure 2. Wave vectors $\vec{k}_{1}$ and $\vec{k}_{2}$ come from one wavefront split into two: one diffracted in direction $\pm \gamma$ with respect to the optical axis $z$, the other has its projection on the plane $x z$ propagating in the direction $\mp \gamma$. Axis $y$ is parallel to the grooves of the nontilted grating $G_{2}$. Angle $\eta$ is the tilt given to grating $G_{1}$. Vectors are decomposed on the nonorthogonal basis formed by the projections of the vectors on the coordinate planes, and-for the sake of simplicity-only some of these components (e.g., $k_{1 x z}$ or $k_{1 x y}$ ) are shown. Vector components $\vec{q}_{x}$ and $\vec{q}_{y}$ are obtained from the difference of the projections of $\vec{k}_{1}$ and $\vec{k}_{2}$ on the $x$ and $y$ axes.

The intensity distribution along the $x$ axis recorded by one row of the detector $(\zeta=$ const.) is given in Figure 3. This figure shows the contribution of each discrete wavefront, but in fact, the interferogram is the combination of the contributions of all wavefronts with varying wave numbers irradiating the detector. The inverse FT of the interferogram given by Equation (7), which is performed numerically with a discrete Fourier transform algorithm, recovers the spectrum $B(\sigma)$.

Equation (6) implies that the interference pattern will have two dimensional Fizeau fringes, that is alternating bright and dark straight bands of equal thickness, which are inclined on the plane of the detector. Depending on the position of the wave number $\sigma$ with respect to $\sigma_{0}$ in the spectral axis, these fringes will be inclined clockwise for redder or counterclockwise for bluer wavefronts for $\eta>0$, or vice versa for $\eta<0$. For the special case $\eta=0$, the fringes produced by the wavefronts with wave 
numbers $\left|\sigma-\sigma_{0}\right|$ will be indistinguishable and all will be vertical (i.e., parallel to the grooves on the gratings).
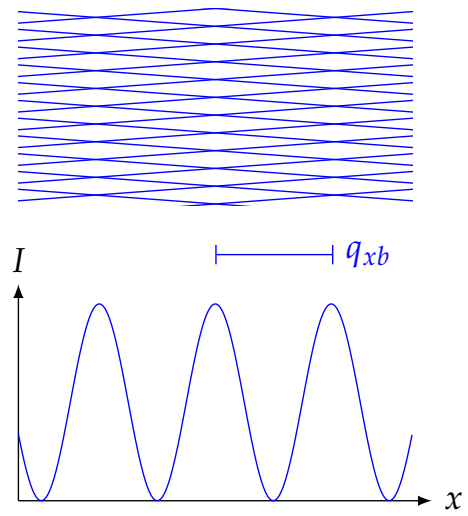
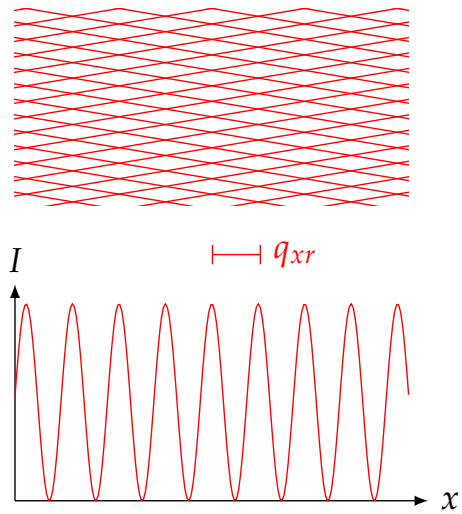

Figure 3. Intensity distributions recorded by a row of the detector array along the $x$ axis for a series of wavefronts with different wave numbers, separated for clarity. Periodicity of the interference fringes for each wavefront is given by $q_{x b}$ and $q_{x r}$. In a measurement, the detector records the sum of intensity distributions for all wavefronts irradiating it.

The interference fringes along the $x$ and $y$ axes have the following spatial periods

$$
q_{x}=\frac{1}{4\left(\sigma-\sigma_{0}\right) \tan \theta}
$$

and

$$
q_{y}=\frac{1}{\sigma \eta}
$$

where $q_{x}=\left|\vec{q}_{x}\right|$ and $q_{y}=\left|\vec{q}_{y}\right|$ are the magnitudes of the projections of vector $\vec{q}=\vec{k}_{1}-\vec{k}_{2}$ into $x$ and $y$ axes respectively, as shown in Figure 2. Since the period along $x$ is heterodyned, it has a much higher resolution as compared to the period along axis $y$. Therefore, in experiments, the angle $\eta$ is used to distinguish the contributions from the wavefronts with wave numbers $\left|\sigma-\sigma_{0}\right|$, effectively doubling the spectral range, but not for spectral analysis. In practice, the latter can be performed only when higher diffraction orders are considered, as in echelle spectrometry.

The recording of the interference fringes is done by an image sensor, which means that $q_{x}$ and $q_{y}$ are sampled by a 2D detector array with $N_{x}$ and $N_{y}$ pixels along each direction, each with square pixel pitch $\ell \times \ell$. Neglecting quantization (conversion of irradiance into digital format), the discretization of $q_{x}$ and $q_{y}$ yields $q_{x}=N_{x} \ell$ and $q_{y}=N_{y} \ell$. Based on the Nyquist-Shannon sampling theorem, the smallest period that can be resolved is twice the pixel pitch, i.e., $q_{\min }=2 \ell$. Consequently, when an imaging system with unit magnification is considered, the largest wave number change $\Delta \sigma=\left|\sigma_{\max }-\sigma_{0}\right|=1 / q_{\min }$ that can be recorded is

$$
\Delta \sigma=\frac{1}{8 \ell \tan \theta} .
$$

This equation determines the unaliased bandwidth of the instrument. When considering the transverse magnification $M$ of the optical system that images the fringes onto the detector, the value for $\ell$ in the above equation should be changed with $M \ell$.

The resolving power of the instrument is determined by the maximal value for $q_{x}$, which corresponds to the diameter $w$ of the overlapping beams with the smallest wave number change $\delta \sigma=\left|\sigma_{\min }-\sigma_{0}\right|$ on the detector, or the aperture of the imaging system. In practice, $w$ is the diameter of the field of view that is imaged onto the detector. Since $\delta \sigma=1 / 4 w \cdot \tan \theta$, the resolving power is 


$$
R=\frac{\sigma_{0}}{\delta \sigma}=4 w \sigma_{0} \cdot \tan \theta .
$$

Setting a measurement with a required $\Delta \sigma$ and $R$ means that one has to adjust the values for $\sigma_{0}$ (or, equivalently, adjust the angle $\theta$ ), and $w$, and then, for a recorded interferogram based on Equation (7), the working equation is

$$
B(\sigma)=\int_{-\infty}^{\infty} I(\xi) \cos 2 \pi \xi\left(\sigma-\sigma_{0}\right) d \xi
$$

where a constant contribution is neglected.

Experimentally, the removal of the constant contribution is done by recording three images under the same conditions: one image is the interferogram, and two other images are recorded by alternatively blocking each grating. Finally, the image that is evaluated is the difference between the interferogram and the sum of the images from each grating. This yields the image of only modulated interference fringes, which is evaluated numerically using a fast Fourier transform (FFT) algorithm. The output of FFT is a matrix of magnitudes, in which a region of interest is defined. This region is determined by calibrating the instrument with a light source of known spectrum. The final spectrum is obtained by summing the magnitude values along the nonheterodyned direction.

\section{Materials and Methods}

\subsection{Rock Samples}

SHS was applied for mineral determination of three rocks of different origins: marble from Carrara, Italy, copper ore from Los Pelambres, Chile, and nickel ore of unknown origin [12]. Samples shown in Figure 4, are cut in the form of sectors and polished. Their composition was determined with a benchtop spectrometer (Horiba Labram HR 800 Raman Spectrometer coupled with an Olympus BX41 upright microscope), which yielded the control dataset. In obtaining these data, an area of $10 \mathrm{~mm} \times 10 \mathrm{~mm}$ was scanned and spectra were recorded in $0.5 \mathrm{~mm}$ steps. Thus, in total 441 spectra per sample were obtained, under the same experimental conditions: the sample was irradiated by a continuous wave (CW) laser with a wavelength of $532 \mathrm{~nm}$ and power of $34 \mathrm{~mW}$ at the sample surface. The laser was focused on the surface of the sample using a $10 \times$ (numerical aperture N.A. $=0.25$ ) microscope objective, and the scattered light was collected in the $180^{\circ}$ backscattering geometry and integrated for one second, dispersed by a $300 \mathrm{gr} / \mathrm{mm}$ grating and detected by a liquid- $\mathrm{N}_{2}$-cooled CCD (charge-coupled device) camera operated at a temperature of $-130{ }^{\circ} \mathrm{C}$ having 1024 pixels along the spectral axis. The resolution of the spectrometer, expressed as the difference in wave numbers between two adjacent pixels, is between 2.5 and $3.7 \mathrm{~cm}^{-1}$, depending on the Raman shift within the acquired spectral range. These control spectra are shown in Figures 5-7. All three figures show the averaged Raman spectrum from all 441 acquisitions. Figure 5 shows the Raman spectrum of calcite with the most prominent Raman bands at $1087 \mathrm{~cm}^{-1}$ and $285 \mathrm{~cm}^{-1}$, as well as bands at $715 \mathrm{~cm}^{-1}$, $1440 \mathrm{~cm}^{-1}$ and $1753 \mathrm{~cm}^{-1}$. The inset shows the Raman band at $1087 \mathrm{~cm}^{-1}$, which-together with the band at $285 \mathrm{~cm}^{-1}$-was recorded in all acquisitions. Two observations then can be made: first, the homogeneity of marble is inferred from the repeatability of the spectra. Furthermore, the collected light from marble did not saturate the detector within the chosen integration time (1 s). Figure 6 shows the average Raman spectrum from copper ore and the inset shows the Raman map of the Raman band at $466 \mathrm{~cm}^{-1}$ for the acquisitions where the signal-to-background-noise ratio $\left(\mathrm{SN}_{b} \mathrm{R}\right)$, which is defined as the ratio between the maximum of the signal and the standard deviation of the part of the spectrum without a signal [13], was larger than 3. The acquisition of Raman spectra from copper ore resulted in about $10 \%$ of the spectra being saturated, and about half being featureless (only fluorescence was recorded). Analysis of the copper ore spectra revealed that quartz is the main constituting mineral because its most prominent band at $466 \mathrm{~cm}^{-1}$ was detected in about 200 spectra. Rutile and albite have also been detected, but only in two and in one spectra, respectively. As for the nickel ore, shown in 
Figure 7, the saturation of the detector was not a serious problem but only a quarter of the acquisitions yielded a detectable Raman signal and all of those belonged to forsterite mineral, inferred from the characteristic doublet at $825 \mathrm{~cm}^{-1}$ and $856 \mathrm{~cm}^{-1}$. The inset shows the map obtained from the Raman band at $825 \mathrm{~cm}^{-1}$ with $\mathrm{SN}_{b} \mathrm{R}>3$. It is to be noted that the assignment of the Raman bands to minerals is done by using the RRUFF database [14].

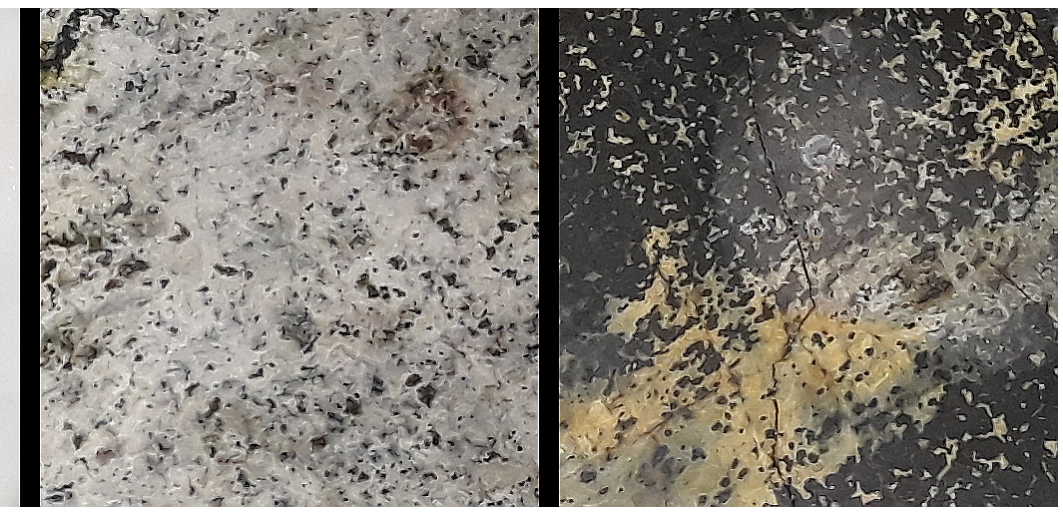

Figure 4. Photograph of the three tested rocks: marble (left), copper ore (center), and nickel ore (right). The dimensions of sample imaged are about $50 \mathrm{~mm} \times 50 \mathrm{~mm}$. Marble has a high degree of homogeneity, while ores show random inhomogeneities in size and distribution.

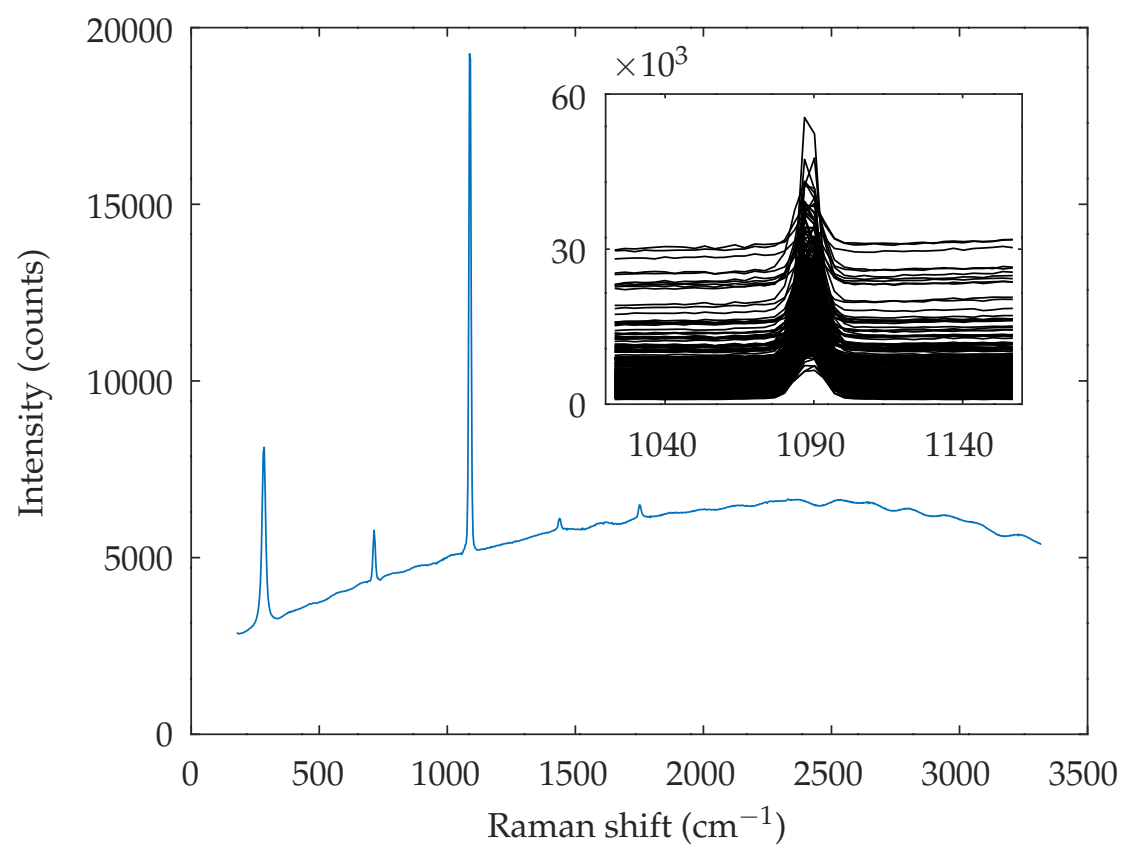

Figure 5. Average Raman spectrum of the control dataset from the marble sample. All 441 acquisitions showed the band at $1087 \mathrm{~cm}^{-1}$ and they are displayed in the inset. 


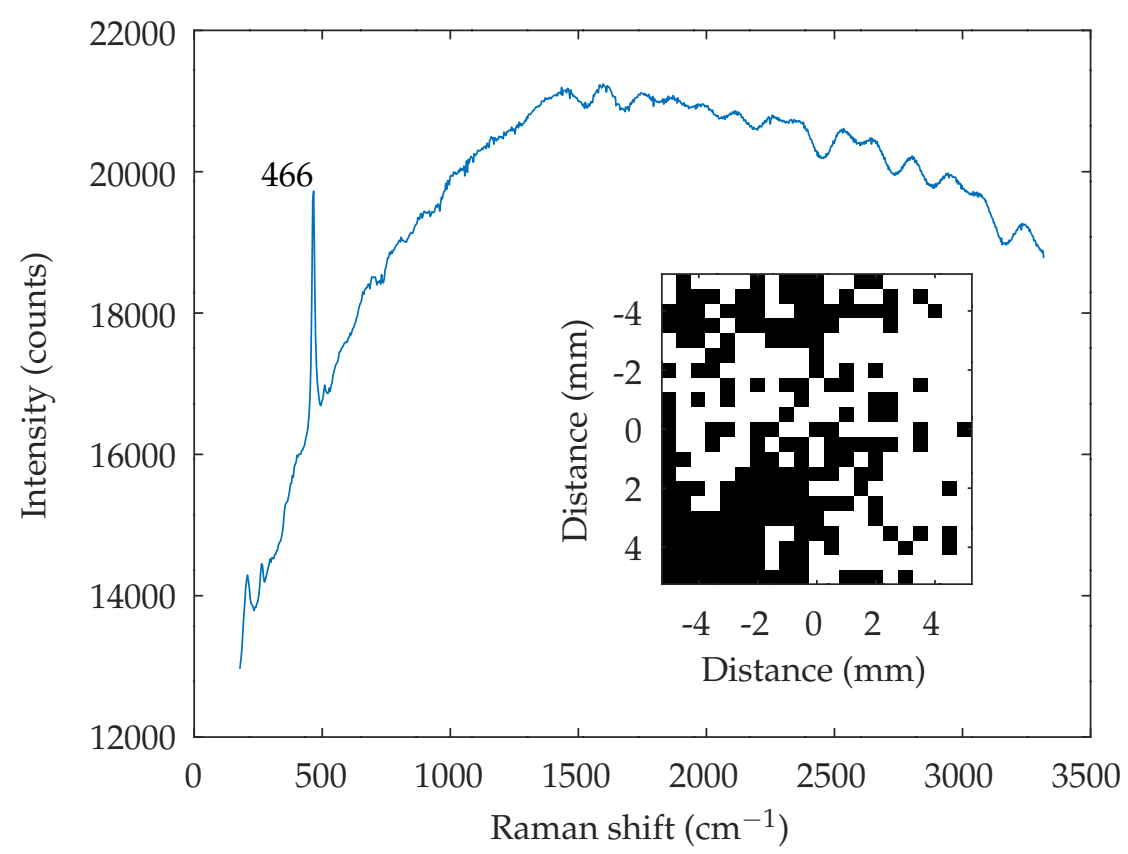

Figure 6. Average Raman spectrum from the copper ore. The inset shows the map of quartz (band at $466 \mathrm{~cm}^{-1}$ ) for which the signal-to-background ratio was larger than 3 . The white dots are locations where the Raman signal of quartz was detected.

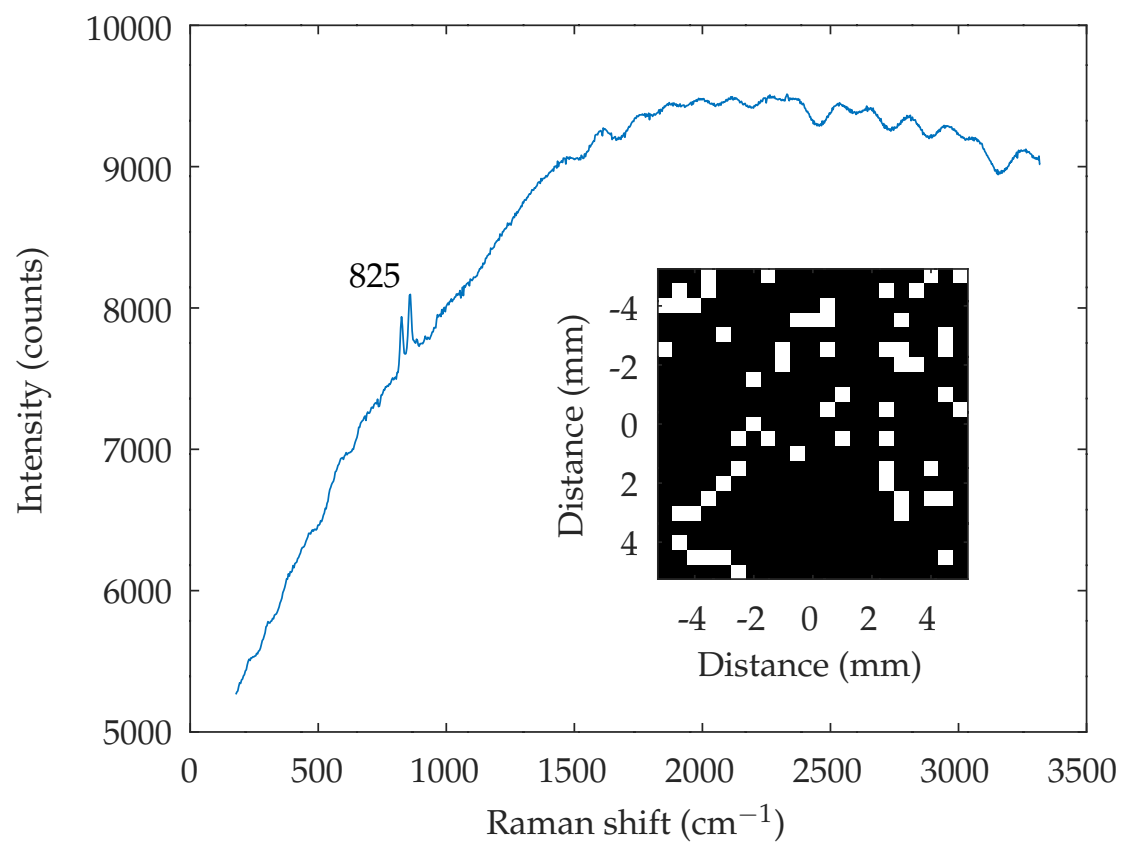

Figure 7. Average Raman spectrum from the nickel ore. The inset shows the map of forsterite (band at $825 \mathrm{~cm}^{-1}$ ) for which the signal-to-background ratio was larger than 3 . The white dots are locations where the Raman band of forsterite was detected.

\subsection{Design of SHR Spectrometer}

The experimental setup for SHRS measurements is shown in Figure 8. Light from a CW diode laser (Green Laser Dot Module) was directed through a dichroic mirror to a $10 \times$ magnification microscope objective, which focused it onto the surface of the sample. The laser power was measured to be $60 \mathrm{~mW}$. The scattered light, including Rayleigh scattering, Raman scattering, as well as fluorescence, was 
collected by the microscope objective $($ N.A. $=0.25)$, guided through the dichroic mirror, which when placed at $45^{\circ}$ reflected all wavelengths lower than $540 \mathrm{~nm}$ and transmitted higher ones, and directed them to the entrance port of the SHS. The optical elements for the SHS instrumentation, i.e., gratings, beamsplitter, lens and the camera, were placed on a breadboard. Their specifications are given in Table 1. A $532 \mathrm{~nm}$ notch filter (OD > 6) and a shortpass filter were placed at the entrance port.

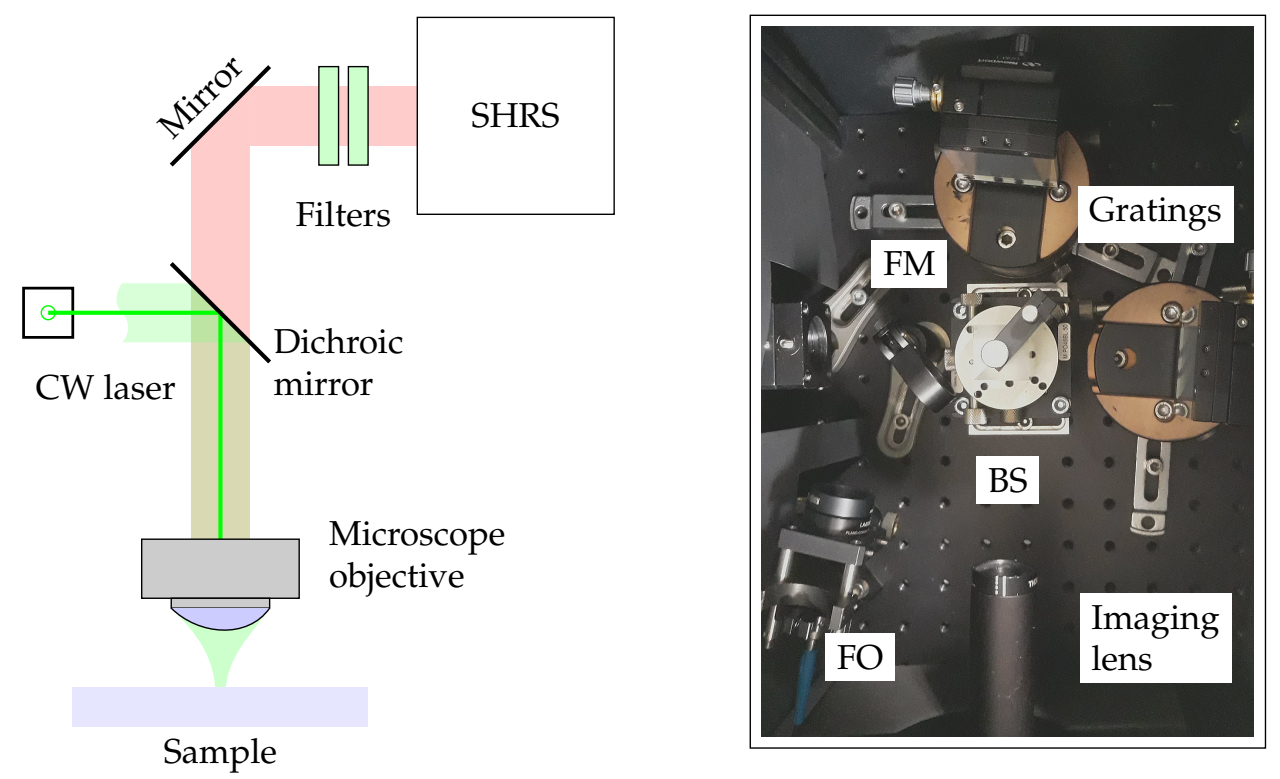

Figure 8. The experimental setup and the instrumentation for spatial heterodyne Raman spectroscopy: FM—folding mirror, FO—fiber optic, BS—-beam splitter. Laser, modular dispersive spectrometer and the camera for SHRS are not shown. The photograph on the right corresponds to the box designated SHRS in the schematic.

Table 1. Optical elements.

\begin{tabular}{ll}
\hline Element & Specification \\
\hline Microscope objective & Thorlabs LMH-10x-532 \\
Gratings & Newport 33010FL01-270R, $50 \times 50 \mathrm{~mm}^{2}, 300 \mathrm{~g} / \mathrm{mm}$ \\
Beamsplitter & Thorlabs BS013, 25 mm \\
Notch filter & Semrock Stopline NF01-532U-25 \\
Dichroic mirror & Semrock RazorEdge LPD02-532R-25 \\
Imaging & CCTV lens, combined with 75 mm focal length 1 inch lens \\
CCD Camera & Retiga R1, 1376 $\times 1024 \mathrm{px}, 6.5 \mu \mathrm{m}$ pixel pitch \\
\hline
\end{tabular}

The limiting aperture of the SHRS setup was that of the microscope objective, which has a diameter of $9 \mathrm{~mm}$. Hence, the field of view that images gratings on the camera was adjusted to be of this size. Since the camera sensor has the area of $6.6 \times 8.9 \mathrm{~mm}^{2}$, the magnification of the imaging system was $\approx 1$. In the first alignment of the SHRS, the gratings were rotated at such an angle $\theta$, so that the wavelength of the laser was close to the starting edge of the spectral range, while the final necessary adjustments were done after the instrument had been calibrated. Since the typical Raman shift for inorganic materials is much less than $3000 \mathrm{~cm}^{-1}$ with respect to the wave number of the exciting laser (in the current study, $\lambda_{\text {laser }}=532 \mathrm{~nm}$, or $\sigma_{\text {laser }}=18,797 \mathrm{~cm}^{-1}$ ), a spectral coverage between 500 and $650 \mathrm{~nm}$ was desirable.

In addition to SHRS, the Raman signal was recorded with a modular dispersive spectrometer, in order to optimize signal recording and directly compare the performance of two compact spectrometers. That is, in the first step, the optimal Raman signal was recorded with the dispersive spectrometer by adjusting the position of the sample using an $X Y Z$ linear motion stage with respect to the static microscope objective, and then, in a second step, a folding mirror on the breadboard 
was folded, allowing for the light to irradiate the beamsplitter and the gratings. For this experiment, Ocean Optics USB2000+ spectrometer was used. This spectrometer has a 2048 pixels linear CCD array detector, and covers the spectral range from 200 to $900 \mathrm{~nm}$. The resolution of the spectrometer was $10 \mathrm{~cm}^{-1}$. The signal was reflected by a folding mirror, focused on the tip of an optic fiber, which was connected to the dispersive spectrometer. The numerical aperture of the fiber and the focusing lens are matched, assuring that the amount of light that enters the dispersive spectrometer is the same as that recorded by the SHRS.

\section{Results and Discussion}

\subsection{Calibration of SHS}

The calibration of the SHS instrument was done by recording the interferogram from a krypton pencil lamp (Newport) to obtain a relationship between the pixel index and the wavelength or the Raman shift, respectively. The obtained interferogram is shown in Figure 9. The image was processed using the FFT algorithm, which gives the image in the frequency domain. As it is known, the FFT produces a mirrored and flipped image, therefore, only a half of the FFT image contains the independent data. Among those, only a narrow strip, which defines the region of interest, contains the desired signal. This region is outlined with red lines in Figure 9, and its inclination is due to the pitch rotation given to one of the gratings. The recovered spectrum shows two lines at noted indices for their respective spatial frequencies. These two lines correspond to the krypton emission lines at $557.03 \mathrm{~nm}$ and $587.09 \mathrm{~nm}$. The Littrow condition of the instrument [15] was determined based on Equation (13),

$$
\lambda_{0}=\frac{f_{2}-f_{1}}{\left(f_{2} / \lambda_{1}\right)-\left(f_{1} / \lambda_{2}\right)},
$$

where $\lambda_{0}$ is the Littrow wavelength, $\lambda_{1,2}$ are the wavelengths of the known source, and $f_{1,2}$ are their respective spatial frequencies. The relationship between the spatial frequency (pixel indices) and the wavelength is then given by

$$
\lambda(f)=\lambda_{0}\left(1-\frac{f \cdot d}{2 \cdot w}\right)^{-1} .
$$

The result of the calibration is that $\theta=5.08^{\circ}$ and $\lambda_{0}=590.19 \mathrm{~nm}$, the spectral range is then from $518 \mathrm{~nm}$ to $686 \mathrm{~nm}$ and the covered Raman shift range is over $4000 \mathrm{~cm}^{-1}$. The imaged part of the grating is calculated to be $w=8.2 \mathrm{~mm}$, which was also confirmed by taking a photo of a ruled paper placed next to the grating and measuring the irradiated area. Hence, the calculated resolving power of the aligned SHRS instrument is $R=4921$. The measured resolving power by fitting a Gaussian to the strongest line, was much smaller and equal to $R_{m}=2935$. There are a few factors that influence the discrepancy between the calculated and measured resolving power: the calculation is done for the ideal case, which assumes perfect collimated beam that enters SHS, and an interferogram without imaging defects. In practice, these conditions cannot be completely fulfilled. The resolution determined from the wave number difference between adjacent pixels was $3.4 \mathrm{~cm}^{-1}$, which is sufficiently fine to be considered high resolution. 


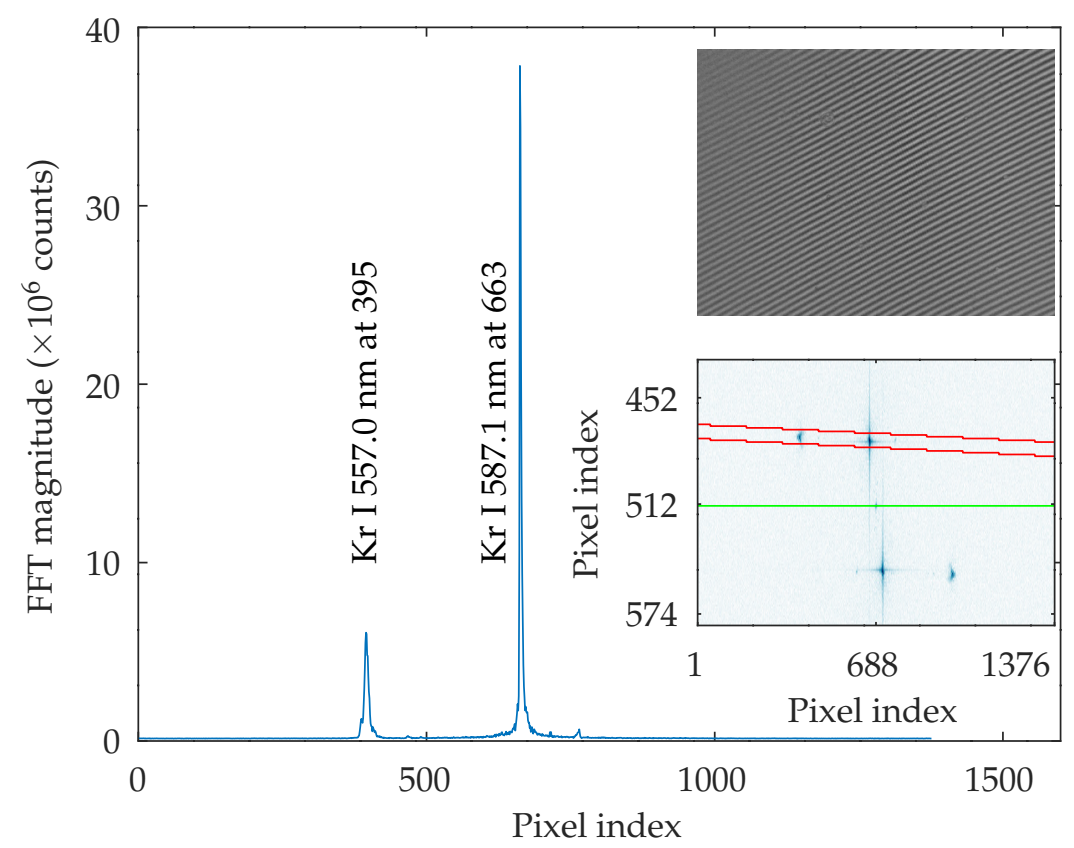

Figure 9. Spectrum of krypton calibration lamp from SHS. The interferogram of the lamp is given in the upper inset, and part of its 2D FFT is given in the lower inset, where the region of interest is defined by the red lines.

The calibration was validated with the Raman signal of sulfur, which is shown in Figure 10. The figure shows the interferogram and the recovered spectrum. The interferogram does not exhibit clearly visible fringes as compared to the case when the calibration lamp was used; nevertheless, upon magnification, fringes can be observed. In addition, the line of zero path difference is visible, as this is the line with the strongest contrast. Interferogram also shows many defects of the imaging optics.

The strong Raman band of sulfur at $473 \mathrm{~cm}^{-1}$ is clearly visible in the recovered spectrum, as well as the band at $865 \mathrm{~cm}^{-1}$. Despite the fact that the laser was filtered by the dichroic mirror and the notch filter, the Rayleigh scattering is observable in the spectrum. The spike and the increased intensity around the Raman shift $2000 \mathrm{~cm}^{-1}$ is due to the defects in the image. Actually, this region corresponds to the zero-frequency region in the frequency domain, therefore, the intensity is increased due to the features in the image that are not repeatable. In particular, the spike at zero-frequency from FFT is quite pronounced and it is easily distinguishable because of its narrow shape; it can be removed numerically in postprocessing. 


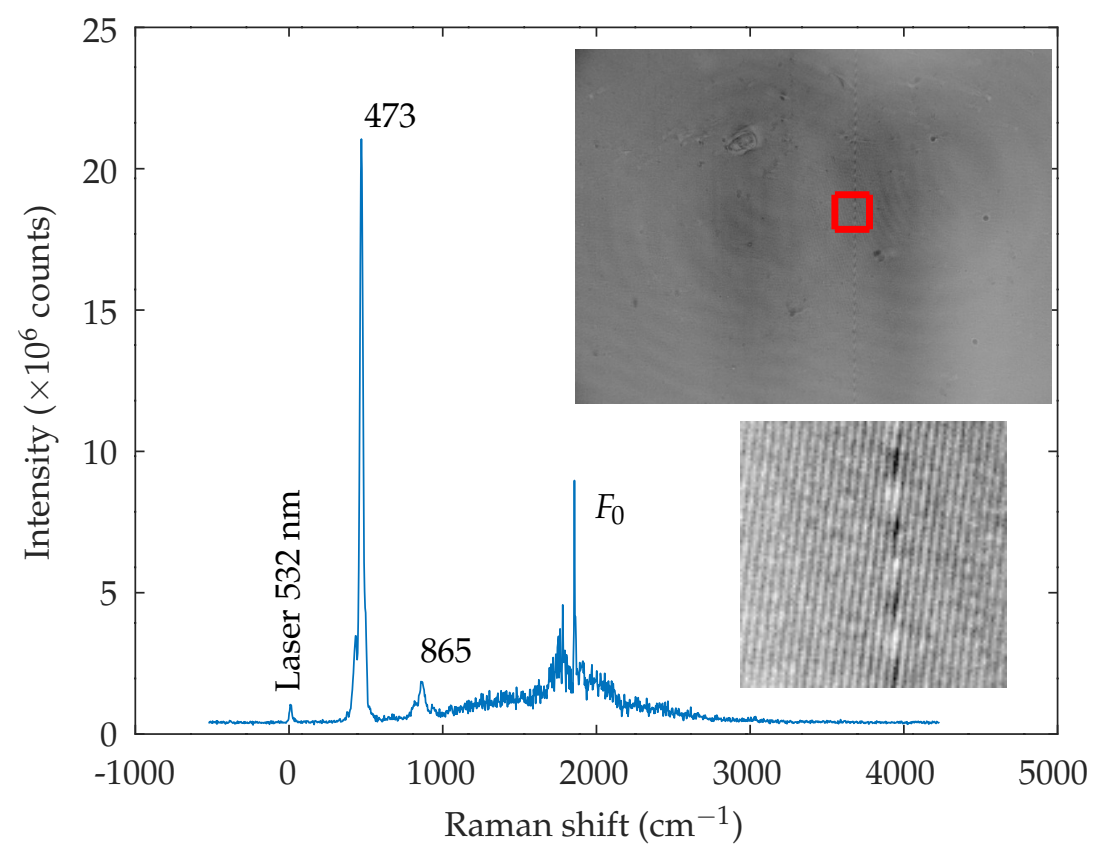

Figure 10. The Raman signal of sulfur. The inset shows the interferogram and a magnified section (outlined with red), where the line of zero path difference is observable. Raman bands of sulfur can be detected at Raman shifts of $473 \mathrm{~cm}^{-1}$ and $865 \mathrm{~cm}^{-1}$. The central part of the spectrum, noted with $F_{0}$, presents the artifacts from the low frequency part of the spectrum, with a narrow and intense spike at the wave number shift equal to the Littrow.

\subsection{SHRS of Rocks}

The determination of minerals constituting the tested rocks was the easiest for marble, as it was expected based on the results of the control dataset. Figure 11 shows the spectra obtained by SHRS and the dispersive spectrometer (Ocean Optics miniature fiber spectrometer). In both cases, the spectra present the average over 10 acquisitions at the same spot, each taken with the exposure time of $10 \mathrm{~s}$. The SHRS recorded two calcite bands, the most prominent one at $1087 \mathrm{~cm}^{-1}$ and a weaker one at $713 \mathrm{~cm}^{-1}$. The first band is due to carbonate ion $\left(\mathrm{CO}_{3}^{2-}\right)$ symmetric stretching and the other is due to asymmetric bending. The latter could not be recorded with the dispersive spectrometer.

The collection of spectra from the other two ore samples was more difficult, mainly due to their weak Raman signals or strong fluorescence. In some acquisitions, even the laser line was recorded despite the fact that it was filtered by the notch filter and the dichroic mirror. Figure 12 shows the Raman spectrum obtained from the copper ore. The Raman band of quartz at $455 \mathrm{~cm}^{-1}$, which is due to $\mathrm{SiO}$ bending, is clearly distinguishable from the SHRS, but not from the dispersive spectrometer. Again, both spectra are the average of 10 acquisitions at the same spot with $10 \mathrm{~s}$ being the exposure time.

One can note two effects on the SHRS spectrum: at higher Raman shifts, the intensity of the spectrum increases, and the noise level becomes larger. Although one might think that the former is due to fluorescence, this is not the case, as this is confirmed by the spectrum from the dispersive spectrometer. The increase is due to the region of the spectrum close to low frequencies (i.e., close to $F_{0}$ ). The increase of noise is also observed in this region. 


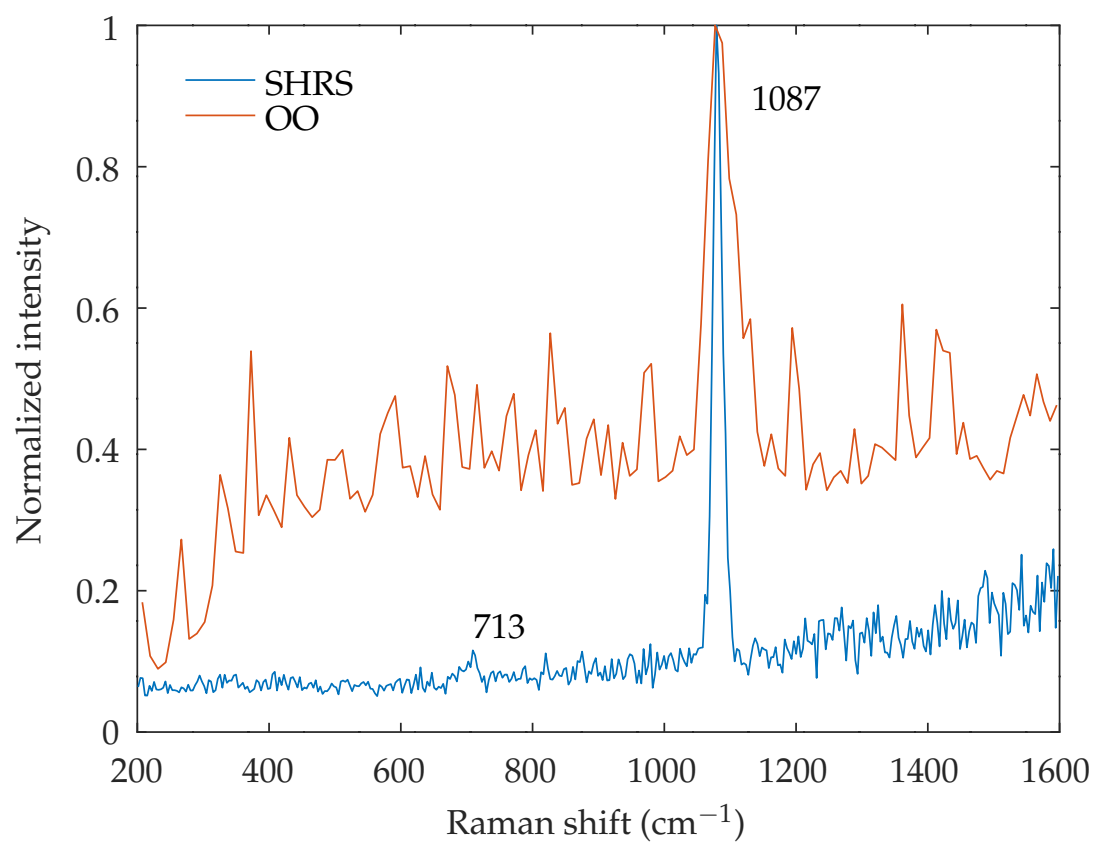

Figure 11. Raman spectrum of calcite $\left(\mathrm{CaCO}_{3}\right)$, obtained from the marble sample. SHRS refers to the spectrum obtained by the SHRS, and OO refers to the spectrum obtained by the dispersive spectrometer (Ocean Optics modular spectrometer). The SNR from SHRS for the bands at 713 and $1087 \mathrm{~cm}^{-1}$ is approximately 10 and 60, respectively. The values for SNR from the dispersive spectrometer were 10 and 50 , respectively.

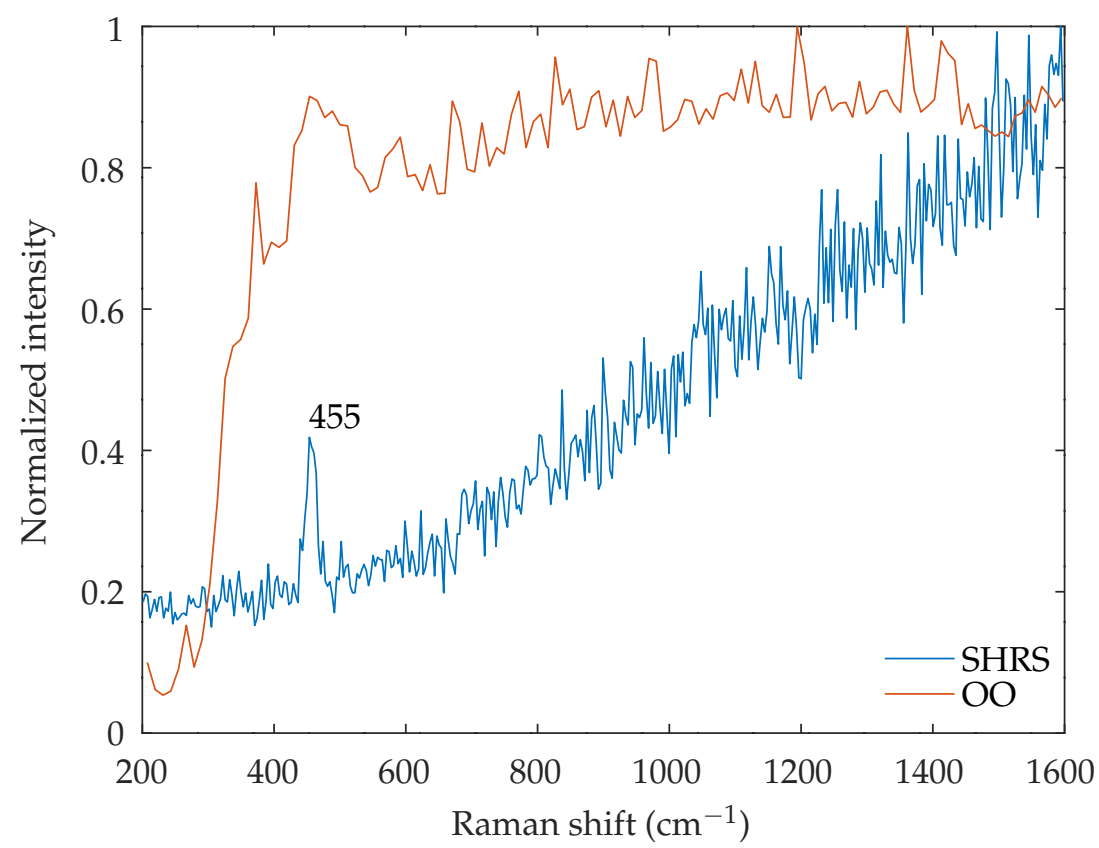

Figure 12. Raman spectrum of quartz $\left(\mathrm{SiO}_{2}\right)$, obtained from the copper ore. The obtained SNR from both SHRS and dispersive spectrometer for the band at $455 \mathrm{~cm}^{-1}$ were approximately 10 .

Figure 13 shows the Raman signal from the nickel ore, which presents the spectrum of forsterite. Similar to the case of the copper ore, here also the increase in intensity is due to the low frequency defects and noise in the signal, and not so much due to fluorescence. The superiority of the resolving power of the SHRS as compared to the grating spectrometer is observed by the separate bands at 
$825 \mathrm{~cm}^{-1}$ and $855 \mathrm{~cm}^{-1}$, which are not observed with the dispersive spectrometer; as a reminder, there are only three pixels in between these bands in the dispersive spectrometer, while SHRS has about eight pixels. These bands are due to strong symmetric stretching of $\mathrm{SiO}_{4}^{4-}$. Although these spectra show the average of 10 acquisitions, now the integration time for the dispersive spectrometer was $1 \mathrm{~s}$, and for the SHRS $10 \mathrm{~s}$. The reason for the lower integration times for the dispersive spectrometer is the need to keep the intensity below the saturation level of the detector.

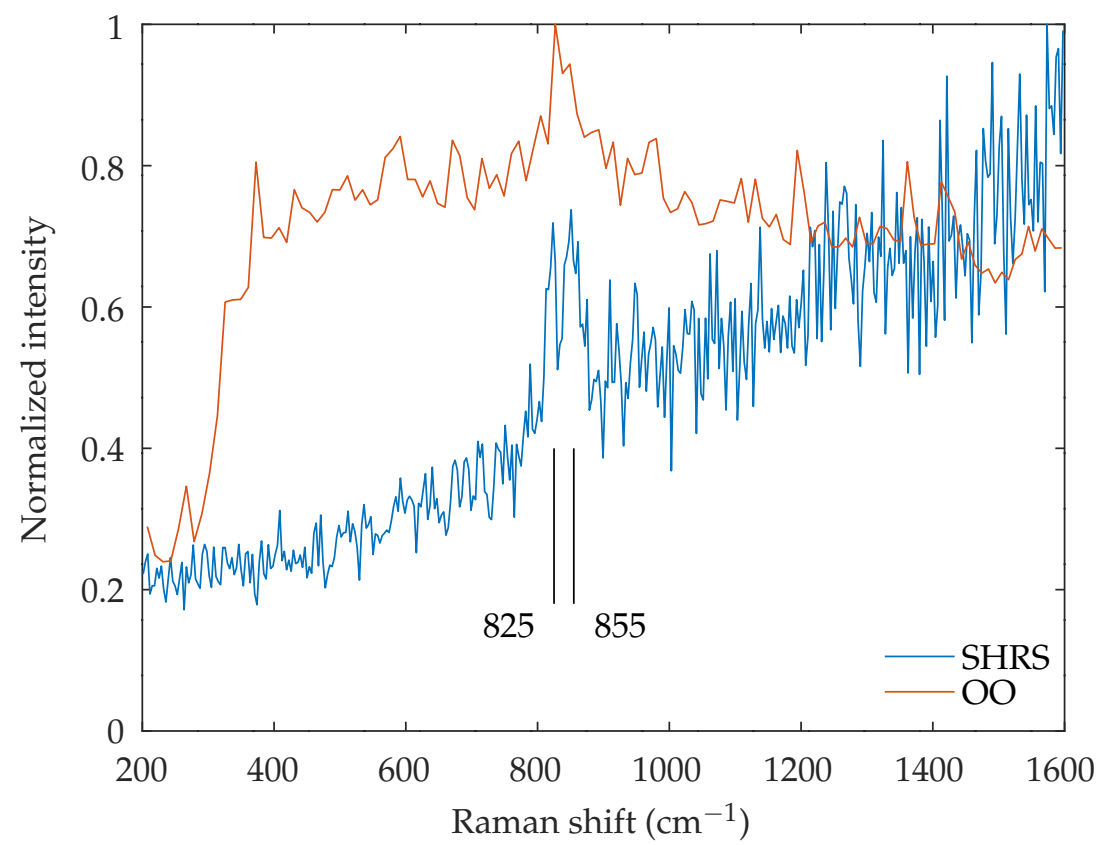

Figure 13. Raman spectrum of forsterite $\left(\mathrm{Mg}_{2} \mathrm{SiO}_{4}\right)$, obtained from the nickel ore. The obtained SNR from SHRS for each band at 825 and $855 \mathrm{~cm}^{-1}$ is approximately 20. These two bands were not resolved by the dispersive spectrometer. The SNR from the dispersive spectrometer for the range covering these bands was approximately 10.

The spectra from dispersive spectrometer in Figures 12 and 13 (lines OO) show elevated background. The sources of this background were the stray laser light, the luminescence and fluorescence of the samples, as well as the optics of the instrument. This noise is detrimental for a qualitative SHRS spectrum, because the SHRS, being an interferometric method, is subject to multiplexing. This means that noise level at each wavelength contributes to the background signal of each pixel. Therefore, the first step in optimizing the SHRS instrument is to reduce and control the level of this noise.

The detection of the signal depends on the level of noise recorded and produced by the instrument. As discussed in the literature (e.g., [16]), the noise distributions in a dispersive spectrometer and in an interferometry-based spectrometer are fundamentally different: for a source dominated by the photon noise governed by Poisson statistics, in the former case the noise is proportional to the square root of the signal at each particular pixel that is recording a certain wavelength, while in the latter case the noise in each pixel is the contribution of the noise from all wavelengths. For this reason, SHS acts as a contrast-enhancing instrument, because the bands with weak intensities are dominated by the noise, while the bands with high intensity benefit from the multiplexing characteristic. Signal-to-noise ratio (SNR) is calculated as the ratio of the average of the intensity at the measured Raman band to the standard deviation of the band intensity and it is presented in the caption of the figures of SHRS spectra. For each case, a series of 10 spectra is used. The comparison of SNR for both spectrometers yielded similar results, which testifies that the spectrum was rather flat, which can mainly be attributed to the fluorescence and luminescence from the source. 
Another issue is the detection of only 1-2 Raman bands in each SHR spectrum. Only some reasons for this are specific to SHRS, as the notch filters used in this version of the setup restrict the Raman shift range to $>400 \mathrm{~cm}^{-1}$ and thus, exclude characteristic low-frequency modes of some minerals (compare the spectrum of quartz in Ref. [6], showing only one band for the same reason). As calcite, quartz, and forsterite are minerals commonly occurring in rocks, the spectra assignment can be considered unambiguous, even if mainly based on the most prominent Raman bands. In the case of calcite, the weak mode at $713 \mathrm{~cm}^{-1}$ enables an undoubted discrimination from other calcium carbonate polymorphs (e.g., aragonite) and carbonates of other elements (e.g., siderite), which exhibit their most prominent Raman mode at a similar wavenumber. In Raman spectra of unexpected and unknown compounds, more bands in each acquisition would be necessary for their identification.

Similar to the collection of the control dataset, a large area of each sample was scanned for Raman signal, yielding close to one hundred spectra per sample, but only a fraction of them yielded useful information. For the marble, the recording of useful Raman signals was achieved in more than $90 \%$ of acquisitions but for the ores this percentage was below $10 \%$ (i.e., out of $80-100$ investigated sample spots, only 5-10 spots yielded Raman spectra). This feature is a serious obstacle when considering the application of SHRS as a handheld device, because it requires scanning a large area of the sample with integration times of the order of seconds, during which time the device must remain stationary. Two adjustments can be used to alleviate this problem: one is to add an imaging system to the SHRS, which would facilitate the correct focusing of the laser onto the surface of the sample. The second adjustment would be the use of a lens with a longer depth of focus instead of the microscope objective, thus allowing for more flexible lens to sample distances.

Rutile and albite, although observed with the benchtop spectrometer, were not observed with SHRS. However, it stands to reason to conclude that this is not due to the performance of SHRS itself, but instead due to the minute amounts of these minerals in the sample. The disadvantage of SHRS compared to the benchtop spectrometer in this respect is the large integration time necessary for the recording of spectra: while a spectrum with the benchtop spectrometer is obtained every second, SHRS requires at least three recordings (interferogram, plus images of each grating), each recording with exposure time of about $10 \mathrm{~s}$.

\section{Conclusions}

An SHRS instrument is assembled and applied for material characterization of three rocks: marble, copper ore and nickel ore. Initially, a set of control data is recorded using a benchtop Raman spectrometer, from where the composition and homogeneity of the samples was determined. Then, the SHRS instrument was used simultaneously with a dispersive spectrometer, which allowed for a direct comparison of the instruments. Both the instruments are of similar size and can be used for field investigations using Raman spectroscopy.

The results from SHRS are promising: the identification of the minerals in nonhomogeneous rocks was achieved with good sensitivity. The performance of SHRS was worse than that of the benchtop spectrometer, but better than that of the dispersive spectrometer, based on the qualitative visibility of the bands on the spectra. Nevertheless, the fact that only a small number of bands is recorded shows that the SHRS needs further adjustments to reach the spectral range with a small Raman shift in order to be applied for unambiguous identification of unknown or multiphase mineral samples. Similarly to the benchtop Raman spectrometer, SHRS detected calcite, quartz and forsterite minerals in the rock samples, although only through the most prominent Raman bands of these minerals, while a couple of minerals, such as rutile and albite, which were observed with the benchtop spectrometer, were not recorded with SHRS. With regards to resolution, SHRS and the benchtop spectrometer yielded similar performance, which was better than that of a dispersive spectrometer. Some steps that can still be undertaken to optimize the instrument, such as better collecting optics, are identified and proposed.

Author Contributions: Supervision and methodology: I.B.G. and T.S.; investigation: A.Z. and A.B.G.; project administration and resources: I.B.G.; software and formal analysis: A.Z. and A.B.G.; validation: T.S.; 
visualization: A.Z. and A.B.G.; writing—original draft: A.Z. and A.B.G.; writing—review and editing: all authors; data curation: A.Z. All authors have read and agreed to the published version of the manuscript.

Funding: This research received no external funding.

Acknowledgments: Authors thank Yi You (BAM Federal Institute of Materials Research and Testing) for lending his personal diode laser. T.S. gratefully acknowledges funding of equipment by DFG GSC 1013 SALSA. Authors thank anonymous reviewers for their insightful comments.

Conflicts of Interest: The authors declare no conflict of interest.

\section{References}

1. Harlander, J.R.; Ronald, J.; Roesler, F.L. Spatial heterodyne spectroscopy for the exploration of diffuse interstellar emission lines at far-ultraviolet wavelengths. Astrophys. J. 1992, 396, 730-740. [CrossRef]

2. Nathaniel, T. Spatial Heterodyne Raman Spectroscopy. Ph.D. Thesis, University of Surrey, Guildford, UK, 2011.

3. Gomer, N.R.; Gordon, C.M.; Lucey, P.; Sharma, S.K.; Carter, J.C.; Angel, S.M. Raman spectroscopy using a spatial heterodyne spectrometer: proof of concept. Appl. Spectrosc. 2011, 65, 849-857. [CrossRef] [PubMed]

4. Egan, M.J.; Angel, S.M.; Sharma, S.K. Standoff spatial heterodyne Raman spectrometer for mineralogical analysis. J. Raman Spectrosc. 2017, 48, 1613-1617. [CrossRef]

5. Lamsal, N.; Sharma, S.K.; Acosta, T.E.; Angel, S.M. Ultraviolet Stand-off Raman Measurements Using a Gated Spatial Heterodyne Raman Spectrometer. Appl. Spectrosc. 2016, 70, 666-675. [CrossRef] [PubMed]

6. Lamsal, N.; Angel, S.M. Performance Assessment of a Plate Beam Splitter for Deep-Ultraviolet Raman Measurements with a Spatial Heterodyne Raman Spectrometer. Appl. Spectrosc. 2017, 71, 1263-1270. [CrossRef] [PubMed]

7. Qiu, J.; Qi, X.; Li, X.; Ma, Z.; Tang, Y.; Mi, X.; Zheng, X.; Zhang, R. Development of a spatial heterodyne Raman spectrometer with echelle-mirror structure. Opt. Express 2018, 26, 11994-12006. [CrossRef] [PubMed]

8. Qiu, J.; Qi, X.; Li, X.; Xu, W.; Zhao, M.; Tang, Y.; Cheng, Y.; Li, W. Raman measurements using a field-widened spatial heterodyne Raman spectrometer. J. Raman Spectrosc. 2019, 50, 1602-1613. [CrossRef]

9. Allen, A.; Waldron, A.; Colon, A.; Raimundo, I.; Sharma, S.K.; Carter, J.C.; Angel, S.M. Remote LIBS, Raman and hyperspectral Raman imaging using a monolithic spatial heterodyne spectrometer. In Proceedings of the FACSS/SCIX International Conference, Palm Springs, CA, USA, 16 October 2019.

10. Palmer, C. Diffraction Grating Handbook, 6th ed.; Newport Coorporation: New York, NY, USA, 2005; Volume 23.

11. Hecht, E. Optics, 4th ed.; Adison Wesley: San Francisco, CA, USA, 2002; pp. 386-389.

12. Hoehse, M.; Mory, D.; Florek, S.; Weritz, F.; Gornushkin, I.; Panne, U. A combined laser-induced breakdown and Raman spectroscopy echelle system from elemental and molecular microanalysis. Spectrochim. Acta Part B 2009 64, 1219-1227. [CrossRef]

13. McCreery, R.L. Raman Spectroscopy for Chemical Analysis; John Wiley \& Sons: New York, NY, USA, 2000; Volume 50.

14. Lafuente, B.; Downs, R.T.; Yang, H.; Stone, N. The power of databases: The RRUFF project. In Highlights in Mineralogical Crystallography; Armbruster, F., Danisi, R.M., Eds.; W. De Gruyter: Berlin, Germany, 2015; pp. 1-30.

15. Englert, C.R.; Harlander, J.M.; Owrutsky, J.C.; Bays, J.T. SHIM-Fire breadboard instrument design, integration, and first measurements. In NRL Memorandum Report; Naval Research Lab: Washington, DC, USA, 2005.

16. Thorne, A. High resolution Fourier transform spectrometry in the visible and ultraviolet regions. J. Anal. At. Spectrom. 1998, 13, 407-411. [CrossRef]

(C) 2020 by the authors. Licensee MDPI, Basel, Switzerland. This article is an open access article distributed under the terms and conditions of the Creative Commons Attribution (CC BY) license (http:/ / creativecommons.org/licenses/by/4.0/). 